\title{
Forum
}

\section{Radiometry and Photometry}

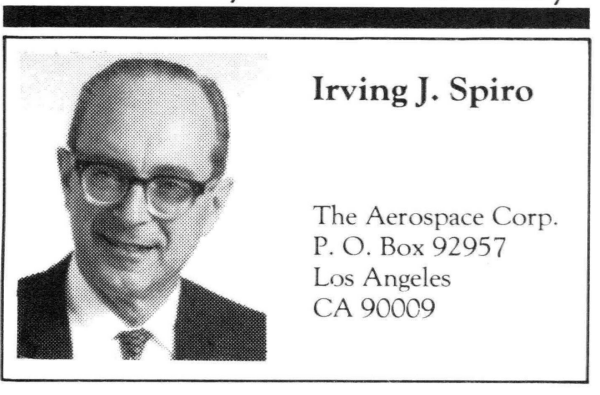

\section{Review of the Sixth (1980) Annual SPIE Seminar on Modern Utilization of Infrared Technology}

My column this month is a review of the sixth annual seminar on Modern Utilization of Infrared Technology, which was held at the Town \& Country Hotel, San Diego, on 31 July to 1 August 1980. The seminar was divided into four sessions:

1. IR Background Suppression Techniques, Irving J. Spiro, The Aerospace Corporation, Chairman.

2. Military Applications/Mosaic Sensor Technology, Col. William Goldberg, Air Force Space Division, Chairman.

3. Military/Scientific Applications, Seymour Konopken, Consultant, R\&D Associates, Chairman.

4. Scientific Applications, Robert A. Weagant, The Aerospace Corporation, Chairman.

In the opening session, a paper on the causes of clutter for earth background staring sensors was explained by E. M. Winter of IBM. He described various sources of clutter and their effects. For example, spatially correlated clutter produces radiance gradients, whereas nonspatially correlated clutter produces white noise that raises the NEFD (and the NET). Interface edges such as ice/water, cloud/desert, and land/ sea produce sharp radiance gradients. He also discussed diurnal effects and shadows.

Theodore Zehnpfennig and his associates from Visidyne disclosed a method of background suppression with variable MTF imaging systems. By "wobbling" or "gyrating" an optical element, a change in MTF is achieved. If the original MTF (A) and the changed MTF (B) have the same slope versus frequency for some range, subtracting B from A will produce a new curve A-B, in which all the frequencies over the common range will produce a zero output, thus suppressing the background in this range.

W. E. Kingsley and W. H. Huber of TRW and A. Lee of Grumman described a packaging technology for a CCD multiplexer with photoconductive detectors. Integrated circuit chips of 32 channels have been made.

T. J. Janssens and S. Valdez of The Aerospace Corporation revealed an optimization program for step-stare infrared sensors. An algorithm that drops out noise frames or noise cells was described. Parameters considered and traded of $f$ included footprint size, diameter of optics, number of detectors, da ta rates, smear reduction, jitter, responsivity, and wavelength.

Tien Tao and his associates from the U.S.Naval Postgraduate School discussed a method of background clutter suppression in which an endto-end signal processing program is used. Professor Tao pointed out that, since the ultimate goal is target recognition, there are five additional steps between background suppression and target isolation. He showed that, by "conventional" adaptive filters, many hundreds of steps are required to converge on a target, whereas by his method of "learning the statistics of an encounter," about 50 steps are required to converge, and by using a Fletcher-Reeves filter, convergence is achieved in 10 steps.

Riley Neel of IBM stated the parameters that must be considered in scheduling a step-stare mission. He considered swath width, the frequency of passing over a particular point on the earth, and system for site selection based on dynamic priority.

Leonard Wald et al. of Lockheed Palo Alto Research Laboratories discussed methods of extraction of targets out of clutter, which is equally applicable to real or simulated backgrounds. Topics covered included spatial, temporal, and spectral filters; centroiding; first, second, and third differencing; drift; edge suppression; and sensor noise.

The second session, devoted to military applications, opened with a presentation by Robert Aguilera of Rockwell International on an advanced infrared imaging seeker program. The seeker now uses a FLIR to acquire a target that is then handed off to the seeker under a method known as "lock-on before launch." A later version will lock-on after launch and seek the target.

Gary Payton et al. of Grumman Aerospace Corporation discussed methods of testing individual sensor strips, which are assembled into modules, which are further assembled into chips. Goals are high yield, high isolation resistance, low crosstalk, high channel uniformity, and high radiometric sensitivity.

Herbert Rauch and O. Firschein of Lockheed Palo Alto Research Laboratories described a mosaic array processor for automatic track assembly. The solution time is independent of the number of tracks. The processor can deal not only with the usual straight line tracks, but also with curved tracks and crossed tracks.

Tom Brown of Honeywell Electro-Optics Center described a 90-element pushbroom image processor that can handle data from $90 \times 512$ pixels and generate hard-copy images or CRT displays. It is being tested under aircraft flightsimulated conditions on outdoor target terrain to provide imagery in the 8 - to $14-\mu \mathrm{m}$ region.

Walter Futterman and R. Benson of Lockheed Palo Alto Research Laboratories described a method for producing simulated backgrounds for use in studies. The output is in $256 \times 256$ pixel form. Eight levels from $0-$ to $12-\mathrm{km}$ altitude have been simulated in SWIR, MWIR, and LWIR spectral ranges and have been catalogued. Both above- and below-the-horizon models are available.

Claus Ludwig of Photon Research Associates described the present state of the knowledge of infrared radiation from rocket plumes. Radiation from plumes is determined with the use of flow, propellant, flow field, radiative transfer, and line-by-line codes. The work uses a six flux model to calculate low-altitude plumes as seen from space. Calculations for data on a global basis remain to be done.

William Malkmus and Newton Freeman of Photon Research Associates and A. Reed of Grumman described a method of practical treatment of particle plumes. Examining the radiation transfer from a plume with scattering and then without scattering results in a differential equation that can be solved. By iteration from the inhomogeneous case to the simpler homogeneous case and repeating, a solution is obtained for plume description at one temperature. They then presented a comparison theoretical treatment versus approximations to obtain a still simpler method.

The session ended with a paper by Jerry Rapier of Rockwell International on clutter leakage approximations for staring mosaic sensors with simultaneous line-of-sight drift and jitter. $\mathrm{He}$ first stated and then described the many parameters that affect clutter. Then with a series of approximations, he presented expressions to illustrate the effects of jitter and drift on each parameter.

The third session opened with a paper by Randall Murphy and his associates from the Air Force Geophysics Laboratory, Boston College, and SSG, Inc., on observations of an F-15 aircraft by BAMM. After stating that BAMM made the first ever measurements with a staring mosaic sensor, they described the goals of BAMM: to measure (or prove the absence of) atmospheric scintillation; and to measure spatial, spectral, and temporal variation of the background. They described the instrument parameters and capabilities and several missions. Data acquired of an F-15 aircraft flying at $50 \mathrm{kft}$ from the balloon platform were presented together with a movie (of the television screen) in which the trail of the aircraft was clearly visible and the birth and growth of a cloud and its shadow were shown.

Professor L. Pau of the French Scientific Mission described a system for detector layout and scanning for an infrared source classification. With the use of an instrument he described the previous year (in SPIE Proceedings Vol. 197), he obtained information in the 3- to 5- and 8to $14-\mu \mathrm{m}$ regions to classify targets. The unit is capable of many scan patterns from individual points and line arrays to circular and mosaic patterns. He showed how the instrument can be utilized to maximize the feature acquisition rate.

Y. Quenneville of the (French) Society for the Application of Liquid Crystals and his associates from Universite Louis Pasteur presented a paper on new infrared imaging system with highresolution liquid crystal sheets. The sheets, which have as many as four different $2 \mathrm{C}$ temperature ranges, are placed on the suspected 
area of a patient and yield temperature information in a few seconds. Pathologies are revealed by relatively large $\Delta t$ 's over a short distance. Sheets of several square meters can be produced.

J. Cunningham and R. Mollicone of Analy tic Decisions, Inc., presented a survey of cryogenic systems for spaceborne infrared applications, capable of 2 to 5 year life and maintaining a system that requires less than 1 watt of cooling a 40 to $170 \mathrm{~K}$. The various mechanical and passive systems were described, and the conditions (temperature, weight, area) for which one would be selected over the other as well as charts of weight per watt were presented.

A companion paper by Tom Nast of Lockheed Palo Alto Research Laboratories discussed the status of solid cryogen coolers. He described the main features such as the need to operate below the triple point of the gas to achieve sublimation. The thermal link to the subject, insulation and vacuum requirements, were discussed. The various gases used and their operating temperature ranges were shown. Of particular interest was a Teal Ruby cooler composed of a methane guard at $60 \mathrm{~K}$ over a neon cooler at $18 \mathrm{~K}$ and a passive radiator. Guards produce increased lifetime for the cooler.

Mike Sentovich of Rockwell International and Mike Haynes of SRI International discussed a paper on aircraft penetration under cloud cover, where observation is defined as a clear line of sight. For a low flying aircraft under cloud cover as reported by ETAC for specific geographic locations, charts for 50 percent and 90 percent observation of the first sighting and the number of sightings in a given period were presented.

The session closed with a presentation by David Murcray of the University of Denver on balloon-borne infrared backgrounds. $\mathrm{He}$ described some missions in Alaska that were flown to verify or disprove the "Murcray" effect. There were some unexplained high signals in the 12 to 16 band obtained on previous measurements in Alaska, but not at Holloman AFB with the same instruments at the same altitude.

On the flights reported here, it was discovered that the nitrogen gas (the exhaust from the $\mathrm{LN}_{2}$ dewar), which was used to cool the entrance optics by flowing over them, caused a cloud or mist to form in front of the window. It was the scattering off of these clouds that caused the high signals associated with the

"Murcray" effect. When the nitrogen flow was stopped, the signals also ceased.

The foregoing explanation, although simple, merely transfers the problem from a radiometer problem to a cloud physics problem, e. g., why does a fog form at altitude due to venting nitrogen in Alaska but not in New Mexico?

The fourth session was opened by C. M. Randall and others from The Aerospace Corporation on the use of a Fabry-Perot etalon for infrared surveillance. A strip burner was used in the laboratory to generate the $\mathrm{H}_{2} \mathrm{O}$ lines. The area under the emission spectra from the water vapor was compared with those of the source as seen through the etalon. The discrimination factor was only 1.1. Although the use of an etalon to distinguish $\mathrm{H}_{2} \mathrm{O}$ in a source was clearly disappointing, the techniques developed may be useful in detecting $\mathrm{CO}_{2}$ or HF. Discrimination factors of about 50 are predicted.

Captain Judd Stailey of the Air Force Air Weather Service described the uses of infrared sensors in meteorology as practiced by both the Air Force and NASA. The use of thermal imagery versus visible images and the information thus obtained were explained. The means of determining temperatures of surfaces and at various locations in the atmosphere were described, and the accuracy obtainable by various methods was emphasized.

Jim Briggs of Boeing proposed the use of a checkerboard pattern in lieu of the familiar tribar as a test target for display evaluation, because it requires less area on the display to obtain the same resolution. He also illustrated how increasing the brightness of the background affects the brightness of the target that may beseen.

Lieutenant Commander Denny Freezer of the Coast Guard Headquarters discussed the evaluation of a variety of sensors in a paper on design considerations for U. S. Coast Guard search and surveillance systems. The system is required to distinguish a man in the water at $1 / 4$ to 1 mile. The only system better than the unaided eye is the FLIR, which also has cost advantages because of the common module hardware in production by the other services.

Ken Cashdollar of the Bureau of Mines described the instruments used for measuring coal dust explosion temperatures. The units must

\section{HIGH RESOLUTION VIDEO DIGIIZING}

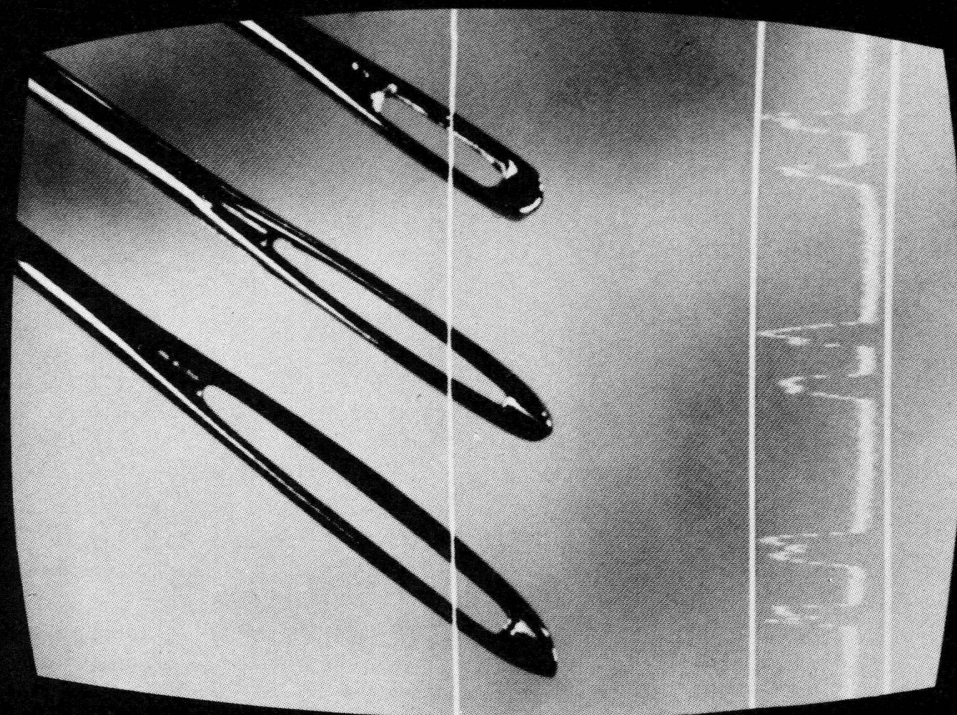

colorado video
Colorado Video's Model 270A-2 is a state-of-the-art TV to computer input instrument designed for use with high resolution TV cameras. Features of the 270A-2 include:

- Computer controllable sampling patterns allowing fast feature identification.

- 1024 x 1024 pixel resolution (optional $512 \times 2048$ ) with 8-bit grayscale.

\section{$\$ 4500$.}

For specifications and applications material, call or write COLORADO VIDEO, 303/444-3972, Box 928, Boulder, CO 80306 U.S.A. 
measure temperatures to $2000 \mathrm{~K}$, be explosion proof, and operate at room temperature. The use of two cube beamsplitters permits the measurement of three wavelength intervals simultaneously.

Ami Ben-Shalom et al. of Technion, Israel Institute of Technology, used various spectrometers to measure the absorption of infrared radiation by atmospheric water vapor. Transmission paths of 1 to $50 \mathrm{~km}$ were used. They found four regions of disagreement with LOWTRAN-4 predictions: at 3.1 7, 4.0, 4.7, and 9 to $12 \mu \mathrm{m}$. For the $3-$ to $5-\mu \mathrm{m}$ region, they deduced that the anamalous absorption results from an incorrect estimate of the amount of water vapor in the sample. By altering LOWTRAN to accept water data in the 3- to $5-\mu \mathrm{m}$ region, there is better agreement with the measured results.

The last paper in the session, by Professor John S. Seeley et al. of the University of Reading (England) described cooled filters, 14- to $42-\mu \mathrm{m}$ wavelength, for infrared astronomy. They have solved the problems of transparency, adhesion, and narrowing of the bulk absorptions useful for long wavelength blocking required to manufacture these filters. Up to 90 layers of $\mathrm{CdSe} / \mathrm{PbTe}$ on $\mathrm{CdTe}$ substrate are used. Professor Seeley stated that their multilayer deposition is comparable to epitaxial layers. Curves were shown for various materials and coatings as functions of wavelength and temperature. We note, with interest, that 4 of the 14 papers presented on the last day were by non-U.S. authors.

/SPIE Proceedings Vol. 253, Modern Utilization of Infrared Technology VI, is available from SPIE, P. O. Box 10, Bellingham, WA 98227. Members \$33, nonmembers \$40. Ed.I

\section{The Business Side of Optics}

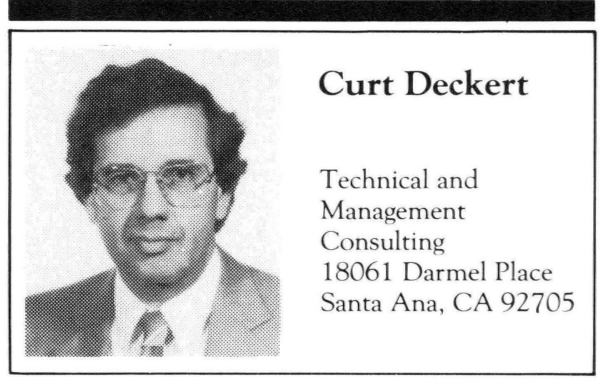

In this issue we consider the economic benefits of reliability engineering. We are happy to welcome Dr. Harry G. Romig to this column. He is a consultant in the area of reliability and has been in that field over a number of years. After receiving his Ph.D. from Columbia, he went on to take significant responsibilities in a number of companies throughout the country. He has later been involved in a number of teaching and consulting activities. He has made a significant contribution to the field of quality control. We are privileged to receive this article from Dr. Romig.

\section{Economic Benefits of Reliability Engineering Harry G. Romig, Ph.D., P.E.}

In today's competitive world market, the U. S. needs to make use of the best available technical knowledge and methods to maximize profits.
However, very often there appears to be a large amount of bias exerted against the use of the techniques developed by quality assurance engineers to make certain that engineering specifications are met and that the ultimate consumer secures a reliable product at a reasonable price.

Products affected include commercial and even those secured by the military under contract. The military often requires multiple bidders for contracts. They also specify in detail what is necessary so that the supplier will provide a product that meets all requirements including the number of hours the product should function in a satisfactory manner prior to failure or its reliability. In commercial markets a guarantee or warranty is often provided the consumer by the supplier. This also requires a measure of reliability.

Suppliers of various types of products and particularly electronic items strive to develop basic reliable designs in conjunction with design and value engineers. New designs must be evaluated by life tests which may require many hours to complete under normal operating conditions. To reduce such testing time, accelerated testing techniques may be used to provide the necessary data. The military were the first to specify conditions under which accelerated tests might be made by providing conditions of test involving specific operating temperatures and vibration for definite periods of time.

One of the most important features of any quality assurance program is the availability of the system to be immediately ready to function when required. For example, in a hospital it is absolutely necessary to have light available at all times for emergency operations. Where there is doubt concerning light or power, a second or third source of energy must be readily available. Also, to provide proper medical aid to patients, medical testing equipment must be available to provide accurate test results. Due to advances in all phases of technology, it is now possible to secure reliable medical testing equipment if it is properly designed with reliable components. Unfortunately, many of the components used are not made under good quality control programs and their Mean Time Before Failure (MTBF) values may range from 50 to 250 hours rather than from 500 to 5,000 (or more) hours. Component parts used for certain types of navigating equipment have MTBF values exceeding 20,000 hours as compared to components used for medical equipment that have a shorter life prior to failure. Thus, to provide reliability and availability, a strict, costly preventive maintenance program must be added for the less reliable medical equipment.

Reliability engineers can reduce costs and increase overall profit if their recommendations are followed. There are usually suppliers who can produce reliable components. The steps to achieve increased reliability goals are presented:

\section{Specifications and Designing for Reliability}

Let's assume that management and marketing used adequate surveys to find products demanded by consumers. From this information, design engineering prepares specifications covering such a product, remembering that reliability must be designed into the final product.

Using easily obtained components, a preliminary breadboard of the product is made. This initial model will give an indication of meeting performance requirements desired by the customer and will demonstrate whether it is possible for such a design to be produced for a competitive price.

At this point, a wise management will have those expert in reliability work with design engineering and determine which are the component parts which might actually fail first. This makes it possible to select final components that will provide the desired life characteristics and operating effectiveness necessary to satisfy consumers. Often at this stage in the product development it will be found that many commercial items will be satisfactory to provide the reliability required. Also, it may be found that the state of the art is such that a few components will never provide the desired reliability so that a change in design is necessary. If a modified design is not feasible, then redundant parts may be provided to improve the reliability, or the product may be designed for very easy replacement of failing parts.

After initial models have been made and tested, a preliminary reliability design review should be scheduled. This review will provide information for the next phase of the operation. What standards may be established for this particular product?

\section{Establishment of Standards}

The preliminary specifications are the basis for establishing standards for a product. Standards must satisfy the consumer, sales, engineering, manufacturing, and management so that the company will be able to make a reasonable profit. One of the most controversial items subject to inspection is one that requires the product to meet the standards of good workmanship. For each such product it is desirable that a sample be made available depicting what is meant by such standards. This avoids accepting inferior quality products where such standards are too lenient.

In particular, finish and color standards samples should be made available for direct comparisons. Also for soldering, welding, polishing, and grinding, samples of acceptable quality should be available for those in manufacturing. Also, specific tolerances that apply are provided on the blueprints and specifications. Overall standards of performance are expressed so as to make certain that the specified quality may be obtained. First articles are checked against such standards before full-scale manufacturing proceeds.

\section{Selection of Supplies of Reliable Components} and Materials

The purchasing departments of most companies select their suppliers by securing competitive bids or may select from a preferred vendors' list. Where military contracts are concerned, great care is taken to have a satisfactory approved vendors' list. However, a better way is to subject products from different vendors to reliability testing in an efficient reliability testing laboratory. This method is very effective in securing quality suppliers because it checks out five or more possible vendors and furnishes the purchasing department with data covering the best suppliers.

Quality industrial concerns maintain complete continuous records of the results of their receiving inspection department while other concerns will have inadequate data. The quality of incoming products will be reflected in the percentage of lots and also units rejected due to poor quality as exemplified by failure to meet acceptance requirements. Based on their past history, vendors may then be classified. The 
purchasing departments may now select the best vendors from this reliability data.

Vendor Evaluations and Receiving Inspection Many companies select and train a team to conduct surveys of vendors to determine whether they are capable of supplying the components desired. Such vendor survey teams may consist of representatives from purchasing, engineering, and quality assurance. Such a survey may be scheduled or may be made by random visits. Better relations are generally secured by arranging for a complete survey in advance so that the vendor may prepare his plant and operations for the quality evaluation survey. If, later, quality seems to worsen, then a random survey might reveal the fact that the supplier is not following his quality manual. When the vendor realizes that he is to be inspected at random, generally he will regain control of his processes and produce quality products.

There are firms that make such vendor surveys and can furnish ratings. Often several companies pool resources and vendor survey data or make a vendor survey jointly. Such practices assure better quality products.

As noted in the last section, the receiving inspection department checks all incoming products to make certain they meet the requirements of the purchase order. Records of these results for each vendor and/or supplier depict the quality of all incoming materials and parts. In the most efficient operations, such results are tied in with inventory control, utilizing one set of operations and efforts. Satisfactory parts, components, and materials are listed in such a way that scheduled operations are recorded so that any products constructed by $\mathrm{R} \& \mathrm{D}$ or by manufacturing will be in line with the specified quality and reliability requirements needed by consumers. Many times such evaluations of quality may be supplemented by somewhat similar overall studies made by expert reliability consultants. Thus the best precepts of value engineering are followed by utilizing the best pertinent information, data, and engineering analyses to provide the most economical product possible.

\section{Engineering and Manufacturing Operations}

A wide spectrum of engineering is necessary to produce modern systems that meet the needs of all kinds of consumers. For example, in the fields of medicine, hospitals and emergency medical clinics need testing systems that provide rapid results to diagnose patients. With the recent improvements in all phases of engineering including lasers and other new light sources, solid state devices, and testing systems, new equipment is becoming available to the medical profession that gives valuable data very quickly, thus obtaining more and better cures and medical services.

As designs are produced by research and development operations, initial models are made to determine the feasibility of the initial design. Improvements are added to these models until the design is finalized. Specifications are prepared and the manufacturing engineers, with the aid of tooling engineers, establish an efficient and continuous manufacturing process. First articles are made and tested at every stage of manufacturing to make certain the production processes are satisfactory. When final approval is obtained for the process, manufacturing proceeds.

\section{Function of Quality Assurance}

Many companies do not bother with a quality

\section{$X$-ray image before processing}

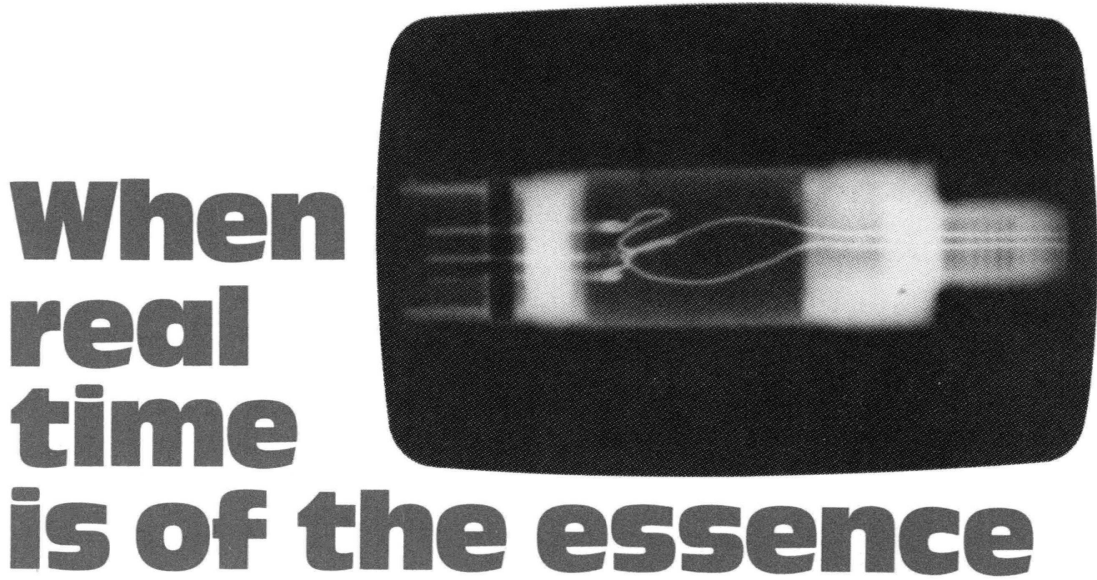

Oucintex Digital Video Processors

In the real world of video processing, images move much faster than traditional A/D converter, computer and software video combinations. Quantex beats the time lag between the video event and pro- $X$-ray image after processing

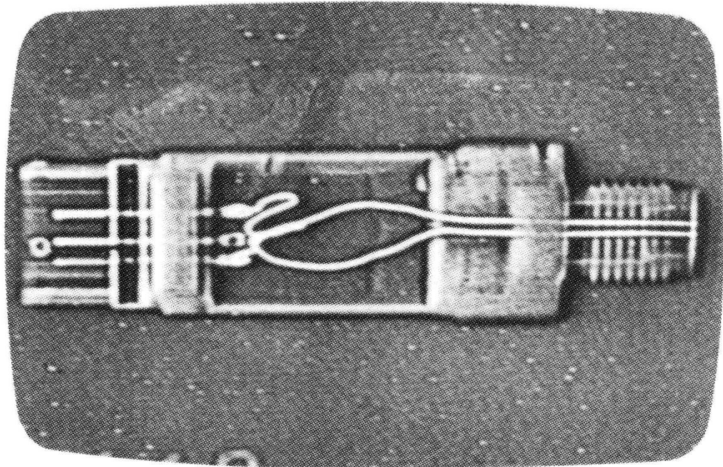
cessed image by simultaneously in putting, computing and outputting, up to 10 million pixels per second transferring an entire picture in $1 / 30$ th of a second

At this real time rate, Quantex digital video processors can:

- reduce noise by summing or averaging frames; as with a time exposure with film,

- eliminate background or compare images to a standard by subtracting frames,

- bring out subtle contrast variations by expanding part of the grey scale,

ənhance edges, and

- store a transient image

Whether you need stand alone performance or pre-processing for quicker data transfer and computer

handling of image data, Quantex lets you instantly improve video quality, process a changing image, eliminate off-line delays, automate imaging, and reduce the use of film. Contact Quantex today for the whole picture.

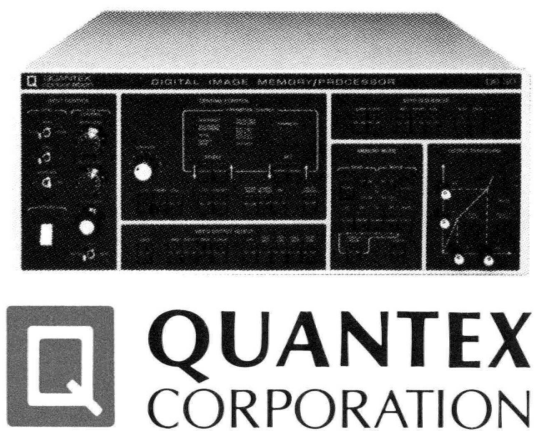

252 No. Wolfe Road

Sunnyvale, CA 94086 • (408) 733-6730 assurance organization. They rely wholly upon inspections to make certain their products meet specifications. This is not as effective and economical as imagined. Later outgoing quality is often studded with defective units which are returned. This results in costly replacements and the development of reputations for giving the consumer poor quality products. A successful quality assurance group, even though small, may be exceedingly helpful in keeping the processes in control.
Quality assurance encompasses both quality control functions and reliability engineering functions. These groups monitor all stages of the entire operation and the important phases of manufacturing, installing control charts where necessary, and stopping production before excessive bad units are produced. Quality assurance also monitors and aids in surveying suppliers so that components received have good reliability. The final result can be excellent products, satisfied customers, and larger profits with 
Your Best Source

FOR

"Off-The-Shelf"

OPTIGS

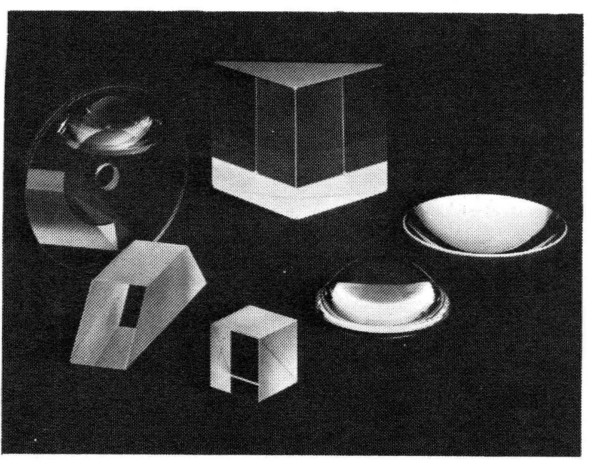

OPTICS FOR INDUSTRY

ROLYN OPTICS 300 North Rolyn Place

P.O. Box 148 - Arcadia, Calif. 91006 (213) 445-6550, (213) 447-3200

effective quality assurance.

Optimizing Design and Manufacturing Cycles

Research and development operations continue the development of related products to provide a complete line of products. Thus there are cycles of operations, including the designs now on the drawing boards and those considered as new products. All phases of the operations including aid from suppliers, consultants, and reliability engineering are carried forward to secure the best possible design for the new products. Additional market surveys are made to make certain that there is a demand for this particular product as designed and at the price which manufacturing has determined can be achieved. Other important information to be obtained from these surveys is the nature of reliability and availability demanded by the specific market target. Controls over such products may also be exerted by consumer or other regulatory organizations in government. Where the product involves medical needs, there may even be restrictions on the nature of the raw materials used in manufacture.

Safety provisions are often required. Caps made for potentially dangerous drugs must be made in such a manner that a youngster cannot open a bottle. Where medical testing equipment may feature the use of various forms of radiation, these must be carefully controlled so that operators of the systems cannot be harmed by even the abnormal use of such equipment.

Assuming one has obtained the optimum de- sign, provisions must be made to apply optimized manufacturing procedures to maximize profits. Manufacturing engineers work with reliability and value engineers to set up manufacturing cycles that provide the best possible product. Where such are purchased from suppliers, the best available vendors are selected and their products checked to determine their life characteristics as well as their adherence to the original design. Thus the complete product is one that may be placed on the market and provide continued profits.

\section{Tests for Reliability}

The application and uses of reliability engineering may prove profitable to many industrial concerns by buying component parts to make certain that the life of their products is beyond their guarantees. In some cases, customers pay more for such reliability data than for the components covered by these life test results.

Where component parts are sold commercially it is difficult to secure life test data from the ultimate consumers. If service contracts are made, customer failure reports may be obtained since elapsed time before service repairs will be recorded. Consumer reports will aid in providing valid life data.

Reliability engineering needs to make adequate test runs prior to the release of the product to consumers. One of the most popular tests is to place 25 to 100 units on test for 1,000 hours and keep records of the number of hours each unit operates prior to failure. For highly reliable parts usually none fail in 1,000 hours, hence, if possible, one should operate the units continuously until all units have failed. In some cases this is not feasible as it may take several years for such a complete test. A compromise time may be achieved by stopping such test after 5 of 100 or $5 \%$ have failed. Then an estimate may be made of the expected life for the other $95 \%$.

Accelerated life test resul ts may be obtained by increasing the severity of the tests by using cycles of heat and cold and adding vibration to the tests. Failures will occur earlier and estimates of reliability under normal conditions of use will be secured from such accelerated life tests which many manufacturers need to maximize overall profits from their products.

Achieving Desired Objectives for Reliability and Profits

Reliability is achieved primarily by providing the best possible design. By making suitable surveys of suppliers, one can make certain that component parts have the desired quality and reliability. Then after assembling the prototype one can determine the reliability of the total product.

Consumers have found in many cases that the practices previously outlined have not been followed and inferior products are placed on the market. Their appearance may be very satisfactory but their performance is often poor. For example, medical testing equipment that will provide excellent test results may have improper components so that they must be continually repaired. Reliability engineers or consultants can help to replace poor quality parts with satisfactory parts and secure reliable products.

Following a program that includes reliability engineering in research and development programs will result in products with greater reliability, thereby providing consumers with their objectives and maximum long term profits for manufacturers.

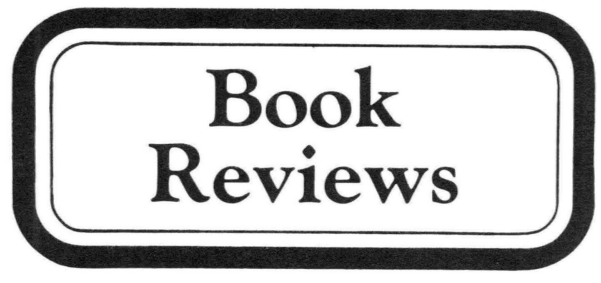

\section{PROCEEDINGS OF THE FOURTH BI- ENNIAL INFRARED INFORMATION EXCHANGE (IRIE)}

Robert A. Miller, Ed. viii +293 pp., profusely illustrated with photographs and drawings (color and black and white), and tables. Seminar held in St. Louis, MO, Aug. 22-24, 1978, under the sponsorship of AGA Corporation, 530 County Avenue, Secaucus, NJ 07094. \$20 complete or $\$ 5 /$ each for four individual sections.

Reviewed by Robert C. Grubinskas, Army Materials and Mechanics Research Center, Nondestructive Evaluation Branch, Watertown, MA 02172 .

The Seminar Proceedings contain 27 technical papers which are grouped into four sections: "Nondestructive Testing/Quality Control," six papers; "Energy Conservation and Plant Maintenance," nine papers; "Research and Development," six papers; and "Instrumentation," six papers.

This particular publication is primarily applications oriented and is concerned with the employment of infrared imaging systems technology to remotely detect, measure, and pictorially display surface temperature variations of objects of interest (thermograms) in both a qualitative and quantitative fashion. Included are papers describing a broad range of thermographic applications conducted in a host of environments-laboratory, industrial, and field. Typical examples are: the thermographic monitoring of damage growth in graphite-epoxy laminates; the use of infrared thermography for industrial heat balance calculations; the thermographic evaluation and heat flow analysis of clothing insulation and garment design; a comparative testing of the applicability of various thermal scanning systems for detecting heat losses in buildings; and the utilization of infrared thermography in air search and rescue missions. The papers describing the employment of digital image processing techniques for infrared thermography are informative and illustrate the progress that is being made to develop the requisite hardware and software.

Overall, the subject publication is a very impressive, well prepared, and superbly packaged product. It contains an abundance of information which would be of interest to anyone who is actively engaged in, contemplating the use of, or just generally inquisitive about the employment of infrared thermographic techniques aa an appropriate means of data acquisition for the fulfillment of specific user needs. The only significant disappointment is the omission of discussion of pyroelectric vidicon technology. Also, in several instances, the labeling of axes associated with empirically determined plots was incomplete in that the units of measurement were not specified. Nevertheless, in the event a decision is made to purchase this publication, it is highly recommended that the complete, rather than the individual-section, option be exercised. This recommendation is made on the basis that 
the existing grouping of papers under the four headings is not unique and could result in not obtaining a desired paper.

For future publications, it is suggested that the numerous appearances of registered trademarks in the text and thermograms be eliminated by defining them only once, and then relegating them in a collective fashion to a single page. It would also be beneficial, from a reader's standpoint, to include an appendix containing the detailed operating specifications of the AGA products that are predominantly described in the technical papers. This information, as currently contained in spearate papers, is both repetitious and incomplete. It would enable a reader unfamiliar with AGA products to better evaluate the technical significance and importance of the embodied papers.

\section{COMPUTER IMAGE PROCESSING AND RECOGNITION}

Ernest L. Hall. 584 pp; b/w and color illustrations; index; bibliography. ISBN 0-12-318850-4 Academic Press, Inc., 111 Fifth Avenue, New York, NY 10003. 1979. \$29.50

Reviewed by O. D. Faugeras, Univ. of Southern California, University Park, Los Angeles, CA 90007.

The book has eight chapters which cover in various depths different areas of the field of digital image processing and analysis. The first six chapters emphasize more the processing part, whereas the last two chapters are devoted to the analysis part. After a short introductory chapter that ends with a useful selected bibliography, the author sets out to describe the perception of images by human observers. Elementary psychophysics and quantitative models of human vision are well covered but very little is said about image formation. Chapter 3 is entitled "Computer Representation of Images" and covers a wide range of topics somewhat superficially: image sampling, quantizing and reconstruction, Inatrix and vector representation, and 2-D transformations. Two welcome innovations are a fairly thorough description of perspective transformations and a short introduction to Number Theoretic Transforms.

Chapter 4 is divided in two parts. The Image Enhancement part covers well the contrast manipulation techniques, various geometric transformations, and enhancement by spatial filtering. A discussion of pseudocolor enhancement techniques can be found in Chapter 5 . The Digital Image Restoration part is more of an introduction to this field and points to the book by Andrews and Hunt, Digital Image Restoration (Prentice-Hall 1977). Chapter 5 is a good description of the theory of the reconstruction of objects from their projections. Examples of the use of these techniques to medical radiography are useful.

Chapter 6 covers the subject of digital image bandwidth compression. The necessary background in information theory is presented first followed by a description of intraframe trans form and predictive coding. Interframe coding is superficially addressed.

Chapter 7 is an introduction to the subject of scene understanding which the author separates further into three areas: segmentation, description of regions, and description of relationships between regions. The discussion of segmentation includes gray level and color thresholding tech-

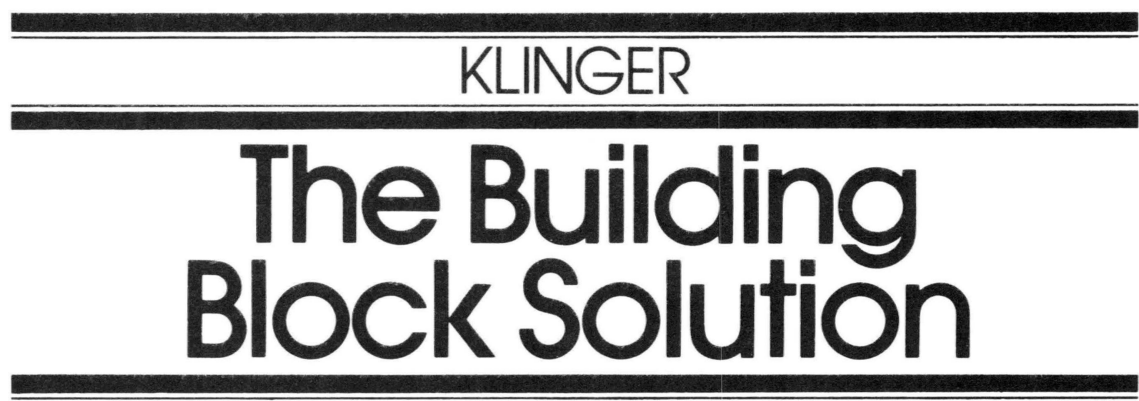

\section{Simplify your Optical/Mechanical Design \& Prołołype Problems with Klinger's High Versatility Building Blocks.}

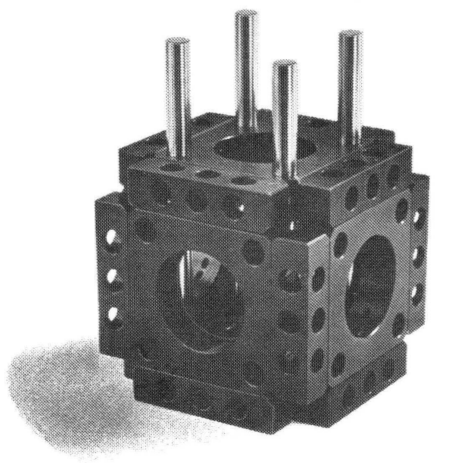

These proven, Micro-Optical Building Block components solve a variety of linear, planar and spatial assembly problems, quickly and economically. Flexible and multi-dimensional, they are used to construct a virtually unlimited number of protoíype, experimental and OEM sub-assemblies.

Choose from a broad selection of structural, interface and active components including support rods, connectors, centering rings and adapters. You may also incorporate lenses, prisms, light sources and other elements to complete your innovative Building Block Solution.
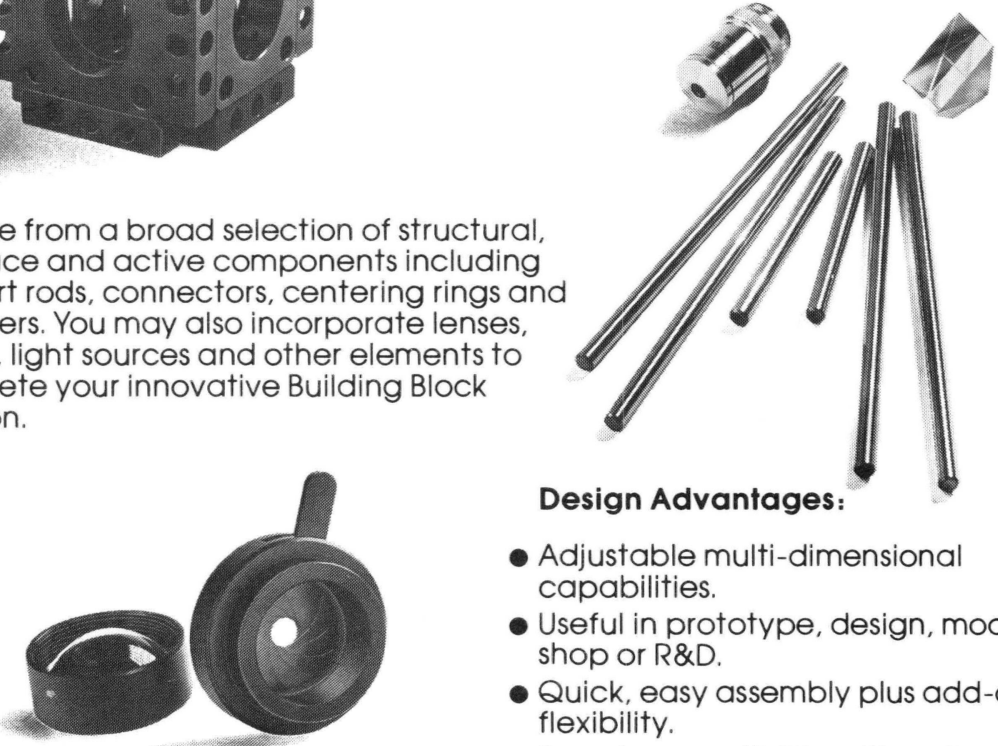

I: KLINGER SCIENTIFIC CORP

Design Advantages:

- Adjustable multi-dimensional capabilities.

- Useful in prototype, design, model shop or R\&D.

- Quick, easy assembly plus add-on flexibility.

- Broad compatibility with optical \& electronic components.

- Durable, sturdy construction, e.g., stainless steel support rods, black anodized adapter plates and connectors.

Write or call (212) 846-3700 for

110-20 Jamaica Avenue

Richmond Hill, N.Y. 11418. (212) 846-3700 
Recognition (Addison Wesley, 1977). Except for the last chapter, the author has tried to cover too many topics, with the result of giving a feeling of superficiality. Nonetheless, I think this should be a useful introductory book for someone not in the field who wants to gain access to more specialized literature.

\section{LASER 79 OPTO-ELECTRONICS}

W. Waidelich, Ed. 662 pp. ISBN 0-86103-020-6. IPC Science \& Technology Press Ltd., P. O. Box 63, Westbury House, Bury Street, Guildford, Surrey GU2 5BH, England.

Reviewed by C. K. N. Patel, Director, Physical Research Laboratory, Bell Laboratories, 600 Mountain Avenue, Murray Hill, NJ 07974.

How does one review conference proceedings, especially when the reviewer did not attend the conference itself? The one advantage of the reviewer not having attended the meeting is that he can afford to give an honest opinion which is not biased by his early commitment to the conference.

But all that aside, the conference proceedings look impressive, both in volume as well as in the authorship of the articles. The conference, Laser 79 Opto-Electronics, appears to have been a very ambitious undertaking encompassing essentially all aspects of lasers and opto-electronics and their applications to a variety of real-life problems, including materials processing, medicine, displays, pollution monitoring, solar cells, laser safety-just to name a few. In other words, the conference has something for everyone and presumably the conference at tracted large numbers of interdisciplinary interest-something that has been lacking in many of the conferences.

In a fast-moving field such as applications of lasers, the conference proceedings will provide a reasonable starting point for both newcomers to a subfield or for an established scientist wishing to broaden his base.

\section{Calendar}

\section{0}

Nov. 18. Symposium on Optical Storage Materials, Yorktown Heights, NY. Co-sponsored by AVS, New York Chapter and SPIE. Dr. T.H. DiStefano, IBM Research Center, Box 218, Yorktown Heights NY 10598.

Dec. 1-3. Topical meeting on Infrared Laser, Lubbock, TX. OSA, 1816 Jefferson PI., N.W., Washington, D.C. 20036

Dec. 8-10. IEEE Intl. Electron Devices Meeting, Washington, D.C. Don Scharfetter, 1980 IEDM Technical Program Chairman, Xerox Palo Alto Research Center, 3333 Coyote Hill Rd., Palo Alto CA 94304. For general information contact M. Widerkehr, Conf. Manager, Courtesy Associates, Inc., 1629 K. St., N.W., Suite 700, Washington, D.C. 20006

\section{1}

Jan. 13-15. Southcon/81 Show \& Convention, Atlanta, GA. Electronic Conventions, Inc., 999 N Sepulveda Blvd., El Segundo CA 90245.
Feb. 9-13. SPIE Los Angeles Technical Symposium, North Hollywood CA. SPIE. Box 10 Bellingham WA 98227. 206/676-3290.

Mar. 22-24. SPIE/ARRS Application of Optical Instrumentation in Medicine IX. San Francisco, CA. SPIE. Box 10, Bellingham WA 98227. 206/676-3290.

Mar. 30-31. SPIE Semiconductor Microlithography VI, San Jose, CA. SPIE, Box 10, Bellingham WA 98227. 206/676-3290.

Apr. 1-2. SPIE Optical Characterization Techniques for Semiconductor Technology, San Jose, CA. SPIE. Box 10, Bellingham WA 98227. $206 \quad 676-3290$.

Apr. 7-10. Ios Alamos Conf. on Optics '81, Los Alamos, CA and Santa Fe, NM. Call for papers in the areas of optical design and evaluation. lasers and laser fusion optics, optical detection. laser and IR spectroscopy, photochemisty, atmosphericastronomical optics, solar optics, biological optics, nonlinear optics, fiber optics, and applications of optics. Poster format. Abstracts due Dec. 1, 1980. Chairman. Los Alamos Conf. on Optic 81. Los Alamos Scientific Laboratory, E-10. MS 430. P.O. Box 1663, Los Alamos NM 87545.

Apr. 7-9. Sira Symposium on Assessment of Imaging Systems, Visible and Infrared, Univ. of Reading, England. Audrey Mills, Conf. and Courses Mgr., Sira Institute l.td. South Hill. Chislehurst. Kent, BR7 5EH. England.

Apr. 20-24. SPIE Technical Symposium East, Washington, D.C. SPIE. Box 10. Bellingham WA 98227. $206 \quad 676-3290$

July 20-24. International Conference on I uminescence, Berlin (West). Sponsored by the Senat of Berlin, the Deutsche Forschungsgemeinschaft, Berlin Physical Society, and the Electrochemical Society. Call for papers. Topics of special interest: material-science and luminescence: nature of luminescence centers (impurities, defects, surface states, etc.): excitons and polaritons: triplet state luminescence: exciplexes: high excitation phenomena and non-linear effects; transient phenomena (picosecond spectroscopy. coherent phenomena, dephasing mechanisms, etc.): coherent processes in the excited state: secondary emission (scattering. resonant fluorescence. hot luminescence): non-radiative processes (multiphonon-processes. auger-effects, collision-free effects): energy transfer: chemiluminescence: luminescence in biological systems: application and device related mechanisms (1.EDs, electroluminescence, cathodoluminescence, displays, laser-devices, solar energy conversion, degradation, luminescence probes). Topics dealing with interactions between organic and inorganic luminescent processes will be favoured. Conference language will be English. Abstracts due Spring 1981. Chairman: I. Broser, Institut für Festkörperphysik II, Technische Universität Berlin, Strasse des 17. Juni 112, Sekr. ER 7, D 1000 Berlin 12.

Aug. 24-28. SPIE 25th Annual Intl. Technical Symposium, San Diego, CA. SPIE, Box 10, Bellingham WA 98227. 206/676-3290.

Aug. 31-Sept. 5. Congress and Twelfth Assembly of the International Commission for Optics, Graz, Austria. Call for papers on astronomical and space optics, unconventional image formation (including optical and hybrid methods, but not purely digital methods), optical materials. Abstracts due March 31, 1981. Conference language will be English. Klaus Schindl, ICO-12. C. Reich- ert Optische Werke AG. Hernalser Hauptstrasse 219. A-1170 VIENNA Austria.

Sep. 7-11. Optics in Biomedical Science Inter. Conf. and Satellite Meeting of the Twelfth Assembly of the International Commission for Optics, Graz, Austria. Call for papers on the biomedical applications of image processing. holography, interferometry, speckle-techniques. spectroscopy, moire-techniques, endoscopy, image formation with non-visible radiation, optical components for improving deficient vision. Abstracts due March 31, 1981. ICO-12 Satellite Meeting. Klaus Schindl, C. Reichert Optische Werke AG. Hernalser Hauptstrasse 219. A-1170 VIENNA Austria.

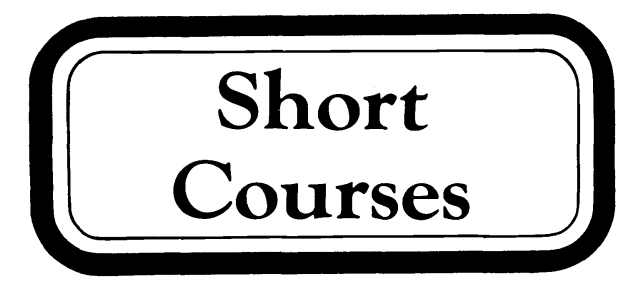

\section{LCI.A Extension course}

Geometric Optics/X437.21 Engineering, Iniv. of California at I.os Angeles, Jan. 6, 1981. $12 \mathrm{mtgs}$. $\$ 180$. Four unit class in design of optical systems. emphasi/ing geometric design aspects. First order or Caussian optics, image formation, third order aberations, prism, and mirrors, spherical and aspheric surfaces, pupils, and optical materials. Application to telescopes, microscopes, photographic lenses. projectors, and other devices. Prerequisite: $B S$ in engineering or science or consent of instructor. Students may register at first class meeting. Text: Warren Smith. Modern Optical Engineering. Contact instructor Milton laikin. 213 640-(0470), or Continuing Education in Engineering and Math, 6266 Boelter Hall. UCl.A. los Angeles (A 90)(24. $213 \quad 825-9971$.

\section{Univ. of California symposium}

"Visibility: An Ipdate of the Field," March 2-6, Scripps Institution of Oceanography, San Diego. $\$ 675$. Designed for researchers and those technical and management personnel responsible for projects involving human visibility and or imaging tasks. Dr. S. Quimby Duntley. former director of the visibility laboratory at Scripps Institution of Oceanography and professor emeritus of the Unis of California, San Diego, and members of the staff of the visibility laboratory will present the program. Marjorie Schneider. UCSD Extension. X-()0l. I a Jolla CA 92093. 714 452-3450.

\section{University of Alabama in Huntsville}

OPTICS COURSES FOR ENGINEERS AND SCIENTISTS. Fundamentals of Optical Science and Engineering, Feb. 2-5, 1981; Instructors, R. Shack, J. Gaskill, W. Wolfe, J. Wyant. Design of Optical Systems, March 2-6, 1981; Instructor, Douglas C. Sinclair. Digital and Optical Image Processing, May 18-21, 1981; Instructors, Edward S. Angel and Brian J. Thompson. For more information, contact Dr. Gary L. Workman, Continuing Education Division, The University of Alabama in Huntsville, Huntsville, AL 35899. Phone 205/895-6010.

SPIE's 25th Annual International Technical Symposium August 24-28, 1981 San Diego 